\title{
Zum Abschluss des Atlasses Ost- und Südosteuropa (AOS). Ein (selbst)kritischer Rückblick des Chefredakteurs
}

\author{
Peter JORDAN, Wien* \\ mit 2 Abb. im Text
}

\section{Konzeption, Organisation und Verlauf des Atlasprojekts}

Das Projekt einer thematischen Kartenserie namens „Atlas Ost- und Südosteuropa“ (AOS) wurde im Jahr 1987 im Auftrag des österreichischen Bundesministeriums für Wissenschaft und Forschung eingeleitet, erschien im Jahr 1989 mit seinen ersten Lieferungen und wurde im Jahr 2014 mit der 30. Lieferung abgeschlossen (vgl. Verzeichnis der Lieferungen im Anhang). Es startete am Österreichischen Ost- und Südosteuropa-Institut als Nachfolgeprojekt des von Josef BREU geleiteten Atlasses der Donauländer und übersiedelte nach der Schließung dieses Instituts im Jahr 2007 an das Institut für Stadt- und Regionalforschung der Österreichischen Akademie der Wissenschaften, wo es die Tradition der dort beheimateten Atlasarbeit (Atlas der Republik Österreich, Niederösterreich-Atlas) fortführte.

Der AOS verstand sich als ein Projekt der Grundlagenforschung mit thematischem Fokus auf den räumlichen Auswirkungen des Transformationsprozesses im östlichen Europa. Dieser thematische Fokus und damit die laufende, diachrone Präsentation wesentlicher raumbezogener aktueller Daten und Forschungsergebnisse waren es auch, die ihn von länderkundlichen Atlanten, darunter auch von seinem Vorläufer, dem Atlas der Donauländer, hauptsächlich unterschieden.

Dieses neue Konzept einer kartographischen Dokumentation aktueller und politisch durchaus relevanter Themen konnte gewagt werden, weil sich im Unterschied zur längsten Zeit des Erscheinens des Atlasses der Donauländer (1970-1989) in vielen Staaten des damals noch kommunistischen Europas - so vor allem in Ungarn und Polen - schon Liberalisierungstendenzen bemerkbar gemacht hatten. So waren nun zu solchen Themen schon Autoren aus diesen Ländern zu gewinnen, Daten und Quellen viel leichter erreichbar geworden. Mit dem politischen Umbruch der Jahre 1989 bis 1991 änderte sich die Situation vollends.

\footnotetext{
* Hofrat Prof. h.c. Univ.-Doz. Dr. Peter JoRDAN, Institut für Stadt- und Regionalforschung, Österreichische Akademie der Wissenschaften, Postgasse 7/4/2,A-1010 Wien; E-Mail: peter.jordan@oeaw.ac.at, http:// www.oeaw.ac.at/isr
} 
Der zusätzliche Wert des AOS im Vergleich mit anderen nationalen und internationalen thematischen Kartenwerken sollte sich aus den folgenden Merkmalen und Publikationszielen ergeben:

- Länderübergreifende Darstellung. Im Unterschied zu nationalen Kartenwerken sollte der AOS thematische Sachverhalte länderübergreifend und damit international vergleichbar darstellen. Dies würde es dem Leser ersparen, nach vielen einzelnen statistischen und kartographischen Quellen zu suchen und die oft unterschiedlichen Klassifikationen und Begriffe miteinander in Beziehung setzen zu müssen.

- Große räumliche Gliederungstiefe. Im Vergleich vor allem mit Welt- und Schulatlanten, aber auch mit dem Europa-Atlas des European Spatial Planning Observation Network (ESPON) sollte sich der AOS relativ großer Maßstäbe bedienen (Hauptkarten im Maßstab 1:3 Mio. und größer) und damit eine wesentlich größere räumliche Gliederungstiefe (zumindest bis zur Ebene NUTS-4) und Detailgenauigkeit erreichen.

- Wissenschaftlichkeit sollte einmal durch große räumliche Gliederungstiefe erzielt werden, wodurch Daten nicht nur im groben regionalen Durchschnitt, sondern räumlich noch differenzierter dargestellt werden können; dann auch mit dem Bemühen, aktuelle Forschungsergebnisse in synthetische, typenbildende Karten umzusetzen und damit mehr als die kartographische Umsetzung von Statistik zu bieten. Als ein weiterer Beitrag zur Wissenschaftlichkeit waren die ausführlichen Begleittexte gedacht, die bei manchen Lieferungen den Umfang wissenschaftlicher Abhandlungen haben und das Kartenbild nicht nur interpretieren, sondern es auch in einen weiteren thematischen Zusammenhang stellen.

- Europa verbinden. Während sich in der Planungs- und Anfangsphase des AOS im westlichen Europa erscheinende thematische Kartenwerke größeren Maßstabs in ihrer detaillierteren Darstellung zumeist auf die Mitgliedsländer der Europäischen Wirtschaftsgemeinschaft (EWG), später der Europäischen Gemeinschaften (EG) und der Europäischen Union (EU) beschränkten - vor allem auch, weil nur für diese einigermaßen einheitlich gesammelte Daten vorlagen -, sollte der AOS jeweils auch die Länder östlich und südöstlich davon gleichwertig in die thematische Darstellung einbeziehen. Damit wollte der AOS Anhaltspunkte für den europäischen Integrationsprozess liefern, an dem die meisten dieser Länder doch sehr interessiert waren und der ja auch vom europäischen Westen intensiv betrieben wurde.

- Verlässliche Quelle der Schreibung geographischer Namen. In der Tradition des Atlasses der Donauländer wollte der AOS besonders auf die korrekte Schreibung geographischer Namen achten und sich dabei nach den Empfehlungen der Vereinten Nationen richten. Der AOS sollte damit als eine verlässliche Quelle für die oft schwer zu ermittelnden und zu schreibenden Namen im östlichen Europa gelten.

- Zweisprachigkeit (Deutsch/Englisch) in Titeln, Kartenlegenden und Begleittexten. Dadurch sollte der Atlas für den internationalen Markt gerüstet und in allen dargestellten Ländern gut verwendbar sein.

Der AOS war als Langfristprojekt eines einzigen Projektträgers organisiert - und nicht, wie heute üblich - als Gemeinschaftsprojekt eines internationalen Konsortiums. Tatsächlich bildete dieser Projektträger aber die Schnittstelle eines internationalen Netzwerks von Forschern und Forschungsinstituten, die zum Atlas entgeltlich oder unentgeltlich beitrugen, sodass der AOS doch als ein internationales Gemeinschaftswerk betrachtet werden kann.

Der AOS wurde wesentlich aus Mitteln des jeweils für die Wissenschaft zuständigen österreichischen Bundesministeriums finanziert. Einige Lieferungen kamen aber auch als kompetitive Forschungsprojekte, die bei Wissenschaftsfonds eingereicht worden waren, oder als Kooperationsprojekte mit anderen Instituten zustande - insbesondere mit dem Leibniz-Institut 
für Länderkunde (IfL) in Leipzig und mit dem Institut für donauschwäbische Geschichte und Landeskunde (IfdGL) in Tübingen.

Die Redaktionsarbeit führte ein kleines Team durch, das aus zwei bis vier Personen bestand, von denen aber zumindest zwei auch noch mit anderen Aufgaben betraut waren. Neben dem Chefredakteur war Florian PARTL die ganze Laufzeit über für den AOS tätig, längere Zeit hindurch waren dies auch Elisabeth Tomasi, Karl Schappelwein und Thede Kahl. Die Redaktion legte die Themen fest und konzipierte gemeinsam mit den zumeist externen Autoren (123 aus 19 Ländern) die Karten und Texte. Sie sammelte in Zusammenarbeit mit diesen die Daten und führte allein die kartographische Umsetzung durch. Sie überwachte die zunächst an das Institut für Kartographie und Reproduktionstechnik der Technischen Universität Wien unter der Leitung von Fritz Kelnhofer und mit maßgeblicher Mitwirkung von Mirjanka Lechthaler, ab der Mitte der 1990er Jahre an die Firma Sféra (Leitung: Oskar Halzl, Eva Hrivnáková) in Pressburg [Bratislava] ausgelagerte EDV-technische Weiterverarbeitung, übernahm sie ab 2009 aber selbst und überwachte ebenso den Druck der Karten und Texte. Manche Karten- und Textmanuskripte erstellten aber auch Mitglieder der Redaktion. Die Auslieferung an die Abonnenten und zum Verkauf über den Buchhandel sowie die Werbung erfolgten in Kommission über den Verlag Gebr. Borntraeger in Stuttgart.

Nach einer im Jahr 1987 beginnenden Planungsphase, an der Fritz KelnHOFER (Institut für Kartographie und Reproduktionstechnik der Technischen Universität Wien) wesentlich mitwirkte, erschienen im Jahr 1989 die ersten beiden Lieferungen, die bei der 13. Internationalen Kartographischen Konferenz (13 ${ }^{\text {th }}$ International Cartographic Conference, ICC) des gleichen Jahres in Budapest vorgestellt wurden.

Das zeitliche Zusammentreffen mit dem Fall des Eisernen Vorhangs war Zufall, dem Interesse am AOS aber sicher förderlich. So erregte insbesondere eine Karte der Umweltprobleme des ganzen östlichen Europas, die als Ergebnis der Arbeit einer Forschungsgruppe des ehemaligen Rates für Gegenseitige Wirtschaftshilfe (RGW) die Umweltsituation dieser Länder zu Ende der kommunistischen Periode vergleichend darstellte und im Jahr 1992 publiziert wurde, großes öffentliches Aufsehen (vgl. Abb. 1).

Neben Umweltthemen erwiesen sich in einer Zeit des gerade im östlichen Europa wieder stark belebten und politisch relevant gewordenen Nationalbewusstseins besonders Lieferungen zum Thema des ethnischen Bewusstseins (als Überblicksdarstellungen des ganzen östlichen Europas, einer größeren Ländergruppe oder als detailreiche Darstellungen der Situation in einzelnen Staaten oder ethnischen Mischgebieten von Staaten) als viel beachtet (vgl. Abb. 2).

Um das Jahr 2000 wurde in Zusammenarbeit mit dem Institut für Geographie und Regionalforschung der Universität Wien und in konkreter Kooperation mit Karel KRIZ, Andreas RIEDL und damaligen Diplomanden des Instituts (Christian FürPaSz, Christian Resch, Robert SAUL) der Prototyp einer interaktiven elektronischen Version des AOS entwickelt und mit einigen Themen (die auch im Druck erschienen waren) belegt. Aus Mangel an finanziellen Mitteln konnte diese funktional sehr leistungsfähige elektronische Version aber nicht mit weiteren Themen bestückt und damit inhaltlich angereichert werden.

Die in den 2000er Jahren schwächer werdende staatliche Unterstützung für ein Forschungsinstitut mit Regionalbezug wie das Österreichische Ost- und Südosteuropa-Institut ließ auch die Erscheinungsfolge des AOS ausdünnen. Sie führte schließlich nach der Schließung des Instituts im Jahr 2007 zu einer Übersiedlung des Atlasprojekts an das Institut für Stadt- und Regionalforschung der Österreichischen Akademie der Wissenschaften, wo es noch einige Jahre - wenn auch mit geringerer Personal- und Finanzausstattung - weiter bestehen konnte. 

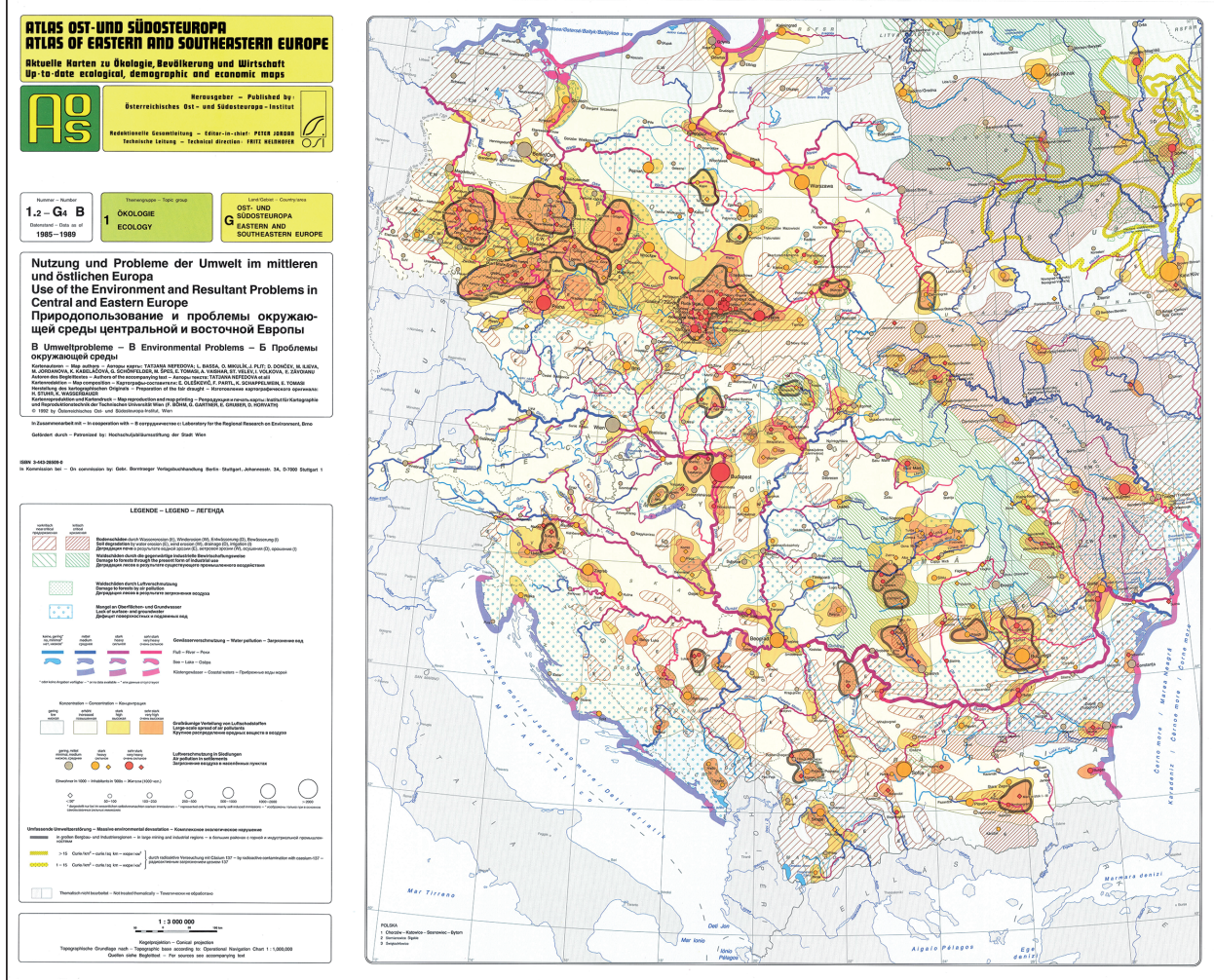

Abb. 1: Die Karte der Nr. 1.2-G4 des AOS zum Thema „Umweltprobleme im mittleren und südöstlichen Europa“. Gebiete umfassender Umweltzerstörung erscheinen in dunklen Flächentönen und mit Bändern umrandet.

\section{Erfahrungen}

\section{Der Atlas der Donauländer war eine wertvolle Schule.}

Die bis zu zehnjährige Mitwirkung einzelner Redaktionsmitglieder des AOS am Vorläuferprojekt „Atlas der Donauländer“ als Kartenautoren und Kartenredakteure war eine gute Schule für Konzeption, Organisation und Ausführung des neuen Atlasses. Dies gilt vor allem für das Bewältigen der großen Problematik des internationalen Vergleichs von Begriffssystemen und Daten, für das Wissen um Datenquellen, das Verständnis für die besonderen kulturellen Merkmale und das politische Beziehungsgeflecht der Region, das „Einlesen“ in deren Sprachen, zum Teil auch deren Erlernen, die Erfordernisse der kartographischen Gestaltung wissenschaftlicher und zugleich gut verständlicher Karten und Legenden und die Prinzipien der Schreibung geographischer Namen in internationalen wissenschaftlichen Kartenwerken. In all diesen Belangen konnte der AOS auf den Erfahrungen beim Atlas der Donauländer aufbauen, ganz besonders auf jenen seines Herausgebers und Chefredakteurs Josef BREU, der sie auch in hervorragender Weise zu vermitteln verstand. 


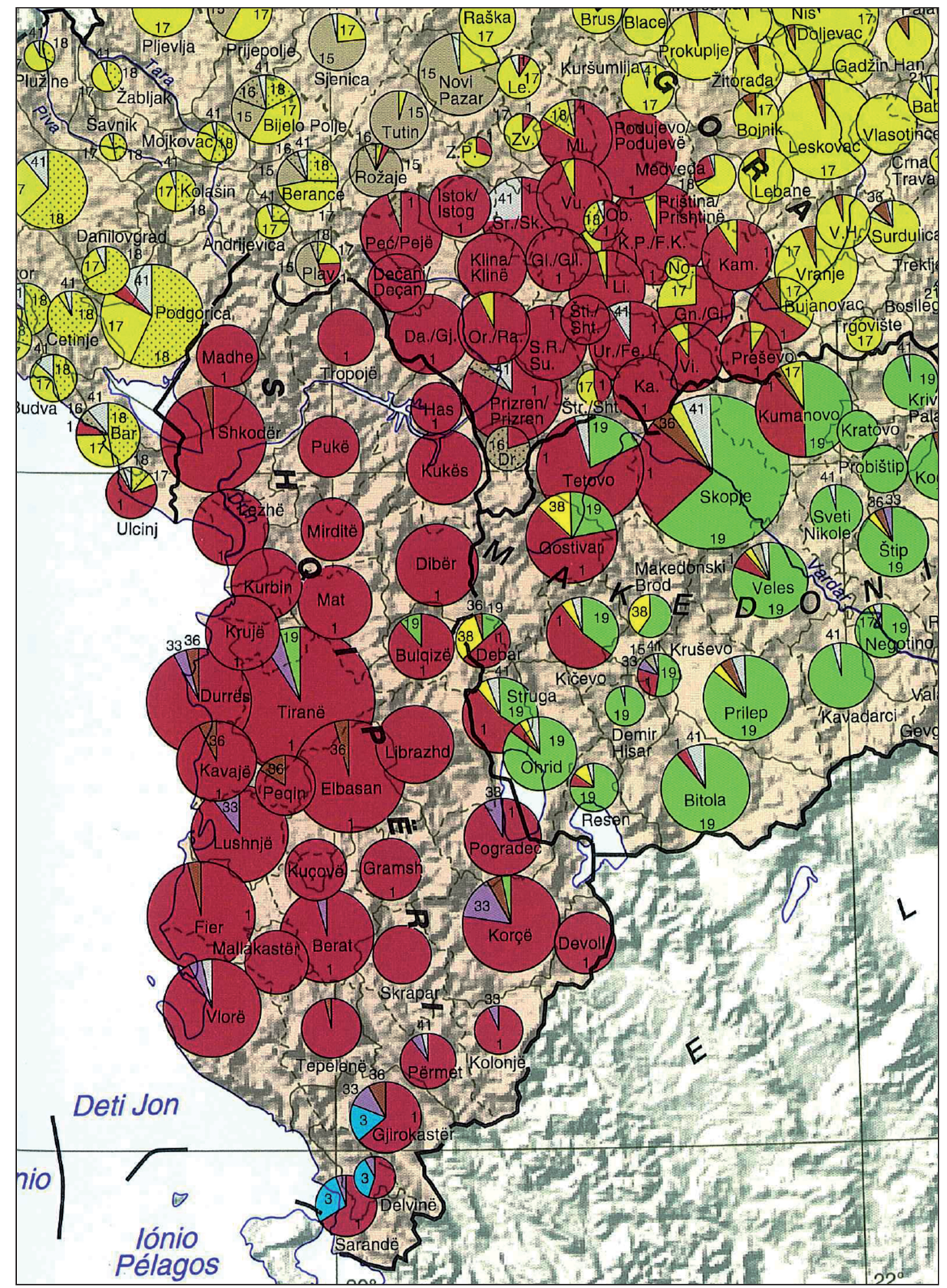

Abb. 2: Ausschnitt aus der Karte der Nr. 2.9-G9 des AOS zum Thema „Ethnisches Bewusstsein in Mittel- und Südosteuropa um 2000“ mit Fokus auf Albanien und albanisch besiedelten Nachbarländern. Die der Bevölkerungszahl einer Verwaltungseinheit proportionalen Diagrammfiguren sind der ethnischen Struktur nach in Kreissektoren gegliedert. Albaner sind durch einen rotbraunen Farbton gekennzeichnet. 
Im Gegensatz dazu waren die wissenschaftlichen Kontakte zu Wissenschaftern und wissenschaftlichen Institutionen, die im Zuge der Arbeit am Atlas der Donauländer natürlich auch aufgebaut worden waren, für den neuen Atlas nur noch sehr teilweise nutzbar. Mit der politischen Wende hatte sich im europäischen Osten auch in dieser Hinsicht ein Umbruch vollzogen: Jüngere, im kommunistischen System noch wenig prominente und jedenfalls nicht mit Kontakten ins westliche Ausland betraute Wissenschafter waren in den Vordergrund getreten und wurden - zumindest schrittweise und nach einiger Zeit - die wichtigen Ansprech- und Kooperationspartner. So entwickelte sich im Laufe der Arbeit am AOS doch ein neues Netzwerk, das infolge der nun ganz anderen politischen Umstände aber viel einfacher, „normaler“ funktionierte und damit auch viel dichter wurde als das des Atlasses der Donauländer.

Expertise über eine Region ist Voraussetzung für die Herausgabe eines wissenschaftlichen Kartenwerks.

Ein wissenschaftliches Projekt über einen Großraum, eben auch ein Atlasprojekt, kann seriös nur betrieben werden, wenn an ihm Experten aus der Region selbst aktiv mitwirken und ihre intime Kenntnis der örtlichen Verhältnisse einbringen sowie ihre leichteren Zugänge zu Daten und Informationen nutzbar machen.

Ein mehrere Länder übergreifendes Projekt bedarf darüber hinaus aber auch der übergeordneten Steuerung sowie externer Sichtweisen. Auch diese setzen Expertise über die Region voraus. Die wiederum kann nur durch fortgesetzte wissenschaftliche Beschäftigung von einzelnen Personen oder - noch besser - ganzer Institutionen mit der Region erreicht werden Spracherwerb eingeschlossen.

Es macht daher durchaus Sinn, wissenschaftliche Einrichtungen zu unterhalten und ihnen eine Basisförderung zuteil werden zu lassen, die sich überwiegend oder ausschließlich mit einem bestimmten Erdraum, einer Kulturregion oder Ländergruppe beschäftigen und in denen sich dazu im Laufe der Zeit Wissen ansammelt. Sehr hilfreich ist dabei Multi- und Interdisziplinarität, denn sie führt zu komplexen Sichtweisen. Der heutige Trend zur Reduktion von Basisförderungen zugunsten der Förderung immer wieder wechselnder Projektgruppen wirkt dem entgegen.

\section{Atlasredaktion ist keine rein kartographische Tätigkeit.}

Selbst wenn von der Redaktion des AOS nicht die Autorenschaft von Karten übernommen worden wäre, sondern sie „nur“ als Kartenredaktion fungiert hätte, wären dazu rein formalkartographische Kenntnisse nicht ausreichend gewesen. Denn es sollen in der wissenschaftlichen Atlasredaktion vorgegebene Sachverhalte nicht nur kartographisch-methodisch korrekt umgesetzt werden; auch ihr fachlicher Inhalt ist systematisch und kritisch zu durchdringen, zu hinterfragen und mit den jeweiligen Autoren zu diskutieren. Nur das gewährleistet eine logisch strukturierte, fachlich einwandfreie und zugleich gut verständliche Legende sowie eine wirklich themengerechte Umsetzung in die kartographische Zeichensprache.

Dazu ist im Falle eines regionalgeographischen Kartenwerks in erster Linie eine gute geographische Ausbildung sehr nützlich. Die heute immer stärkere Entkoppelung von kartographischer und geographischer Ausbildung ist dem nicht förderlich. Die Kartographie versteht sich zu Recht als Teilgebiet der Allgemeinen Zeichentheorie (Semiotik) und damit als eine eigene Wissenschaft, die sich mit den formalen Aspekten der Umsetzung von fachlichen Begriffssystemen in die kartographische Zeichensprache beschäftigt und somit die Inhalte kartographischer Darstellungen selbst nicht in Betracht zieht. Doch erfordert die Praxis der Kartenredaktion vertiefte Auseinandersetzung mit dem jeweiligen Kartenthema, ohne die eine wissenschaftlich adäquate und zugleich gut verständliche kartographische Umsetzung nicht gelingen kann. Es 
ist daher jedem formalwissenschaftlich ausgebildeten Kartographen anzuraten, sich auch um geographische Ausbildung zu bemühen.

Ein thematischer Atlas ist eine gute Grundlage weiterer raumbezogener Forschung.

Mit jeder Karte eines größermaßstäbigen wissenschaftlichen Atlasses - auch mit sehr simpel erscheinenden elementar-analytischen Karten auf der Basis rein statistischer Quellen - arbeitet sich der Autor umfassend in das betreffende Themenfeld und die jeweils dargestellte Region ein. Denn die letztlich in der publizierten Karte sichtbare Information ist nur das komprimierte, nivellierte und zumeist stark generalisierte Kondensat eines Selektionsprozesses aus einer viel umfangreicheren Daten- und Informationssammlung. Diese kann dem Autor eine wertvolle Grundlage für weitere geographische Forschung in diesem Bereich sein. Der Autor der Karte war, um die Daten kartographisch darstellen zu können, auch gezwungen, sie systematisch, vollständig und flächendeckend zu sammeln und aufzubereiten; er ist dabei oft auf Forschungslücken und interessante Forschungsfragen gestoßen, die ihn zur weiteren und noch tieferen Beschäftigung mit dem Thema stimulieren.

So wurde der Verfasser dieses Beitrags nicht nur als Autor, sondern auch als Redakteur von Karten - und nicht erst beim AOS, sondern auch schon beim Atlas der Donauländer - immer wieder in neue Forschungsthemen förmlich „hineingezogen“, für die jeweils aufgrund der für die Karte notwendigen Recherche eine sehr gute Informations- und Datenbasis gegeben war.

Aber nicht nur der Autor der Karte selbst, auch der fachbezogene Leser einer Karte, findet in ihr räumlich gut aufbereitete und - im Falle vieler Karten des AOS - einen ganzen Großraum deckende Informationen als Grundlage und Anregung für weitere Forschung vor.

\section{Ein wissenschaftlicher Atlas hat einen eingeschränkten Nutzerkreis.}

Die in der Konzeptionsphase des AOS gehegte Hoffnung, dass der freilich sehr wissenschaftlich gestaltete und in erster Linie auf ein wissenschaftliches Publikum ausgerichtete Atlas auch im Bereich des allgemeinbildenden höheren Schulwesens oder bei einer interessierten Öffentlichkeit Verbreitung finden werde, hat sich nicht erfüllt. Die Abonnenten des AOS blieben auf Wissenschafter, wissenschaftliche Einrichtungen und Bibliotheken beschränkt - allerdings in weiter internationaler Streuung; dies, obwohl die Öffnung der Grenzen zum europäischen Osten und der Transformationsprozess dieser Länder zumindest in den 1990er Jahren für Viele interessante und viel diskutierte Themen waren. Offensichtich ist Kartennutzung doch keine so allgemein verbreitete Kulturtechnik wie Rechnen, Schreiben und Lesen und bedarf es für jede Nutzergruppe von Karten einer spezifischen, auf diese Gruppe abgestimmten kartographischen Gestaltung.

Wenngleich sich mit dem AOS also nicht alle Hoffnungen erfüllt haben, so öffnete er doch ein weiteres Fenster in einen Kulturraum, mit dem uns in Österreich sehr viel verbindet. Er entspricht auch einer Rolle, die Wien und Österreich hinsichtlich dieses Kulturraums gut ansteht.

\section{Literatur über den AOS}

Hofmayer A. (1991), Der „Atlas Ost- und Südosteuropa“ - eine neue Kategorie internationaler kartographischer Information? In: Österreichische Osthefte, 33, 1. S. 127-146.

Fürpasz Ch., Riedl A., Jordan P., Kriz K., Partl F., Resch Ch., Saul R. (2001), Suitability of a Map Server from a Cartographic Perspective. In: YANG K. (Hrsg.), Proceedings of 
the $20^{\text {th }}$ International Cartographic Conference, ICC 2001 Beijing, China, August 6-10, 2001, S. 2371-2379. Beijing, ICA.

JoRDAn P. (1989), Eine Karte über den Boom des Westtourismus nach Ungarn am Beginn des neuen Atlas Ost- und Südosteuropa. In: GW-Unterricht, 36, S. 17-19.

JoRDAN P. (1990), Aktuelle Karten über den ländlichen Raum in Rumänien im neuen Atlas Ost- und Südosteuropa. In: Geographischer Jahresbericht aus Österreich, XLVII (1988), S. 7-41.

Jordan P. (1990), Der neue Atlas Ost- und Südosteuropa. In: Grundlagen der Weiterbildung Zeitschrift, 1, 2, S. 104-105.

JoRdAn P. (1990), Der neue Atlas Ost- und Südosteuropa. In: Österreich in Geschichte und Literatur mit Geographie, 34, 1, S. 55-56.

Jordan P. (1992), A Series of Thematic Maps on Eastern and Southeastern Europe - A New Breed of Atlases of a Larger Geographic Region. In: Internationales Jahrbuch für Kartographie, 30, S. 63-69.

Jordan P. (1992), Umweltnutzung und Umweltprobleme in Mittel-, Ost- und Südosteuropa. Zwei Karten im Atlas Ost- und Südosteuropa. In: Informationsveranstaltung über umweltrelevante Fragen. Umweltschutzkonferenz im Rahmen des österreich-ungarischen Regionalen Forums, S. 57-71 mit 2 farbigen Kartenbeilagen. Wien - Seibersdorf.

Jordan P. (1993), Editing an international map series - specifics and problems. In: Mesenburg P. (Hrsg.), Proceedings, Vol. 1 of the $16^{\text {th }}$ International Cartographic Conference, Cologne/ Köln, 3-9 May 1993, S. 273-282. Bielefeld.

JoRDAN P. (1993), Novijat atlas na iztočna i jugoistočna Evropa - primer za meždunarodno kooperirane v naučno-izsledovatelskata dejnost [Der neue Atlas Ost- und Südosteuropa - ein Beispiel der internationalen Zusammenarbeit im wissenschaftlich-publizistischen Bereich]. In: Problemi na geografijata, 4. S. 61-67.

JoRDAN P. (1994), Die redaktionelle Arbeit an einer internationalen Kartenserie. Besonderheiten und Probleme. In: Kartographische Nachrichten, 44, 3, S. 96-104.

JoRDAN P. (1994), Small-scale Environmental Mapping as a Tool of Environmental Management and Policy. In: Fodor I., Walker G.P. (Hrsg.), Environmental Policy and Practice in Eastern and Western Europe, S. 287-294. Pécs.

JoRdAn P. (1995/96), Romania in the Scope of Transformation in Central, Eastern and Southeastern Europe. Issues of a map in the Atlas of Eastern and Southeastern Europe. In: Revista geografică, II/III, S. 105-108.

Jordan P. (1996), Anthropogene Veränderungen im Lebensraum der mittel-, südost- und osteuropäischen Länder und ihre kartographische Darstellung. In: Acta Facultatis Rerum Naturalium Universitatis Ostraviensis, 157, 4, S. 145-162.

JoRDAN P. (1996), A Map of International Tourist Attractions in Central and Southeastern Europe with Reference to Croatia. In: Tourism and Hospitality Management, 2, 1, S. 33-39.

Jordan P. (1997), A Map of International Tourist Attractions in Central and Southeastern Europe - Concepts and Methods. In: Department of Regional and Tourism Geography WrocŁaw University (Hrsg.), Conditions of the Foreign Tourism Development in Central and Eastern Europe, Bd. 4, S. 73-80. Wrocław.

JoRDAN P. (1997), Toponymische Redaktion von Kartenwerken am Beispiel des Atlasses Ostund Südosteuropa. In: Kretschmer I., Desoye H., Kriz K. (Hrsg.), Kartographie und Namenstandardisierung. Tagungsband zum „Symposium über geographische Namen“ (= Wiener Schriften zur Geographie und Kartographie, 10), S. 79-85 mit einer Karte als Beilage. Wien, Institut für Geographie der Universität Wien, Ordinariat für Geographie und Kartographie. 
JoRDAN P. (1998), The „Atlas of Eastern and Southeastern Europe“ as a New Device of Cartographic Information on Eastern Europe. In: Papers, "100 Anniversary of Geography at Sofia University, International Scientific Conference, Sofia, 14-16 May 1998, S. 334-342.

JoRDAN P. (1999), Die touristische Attraktivität des östlichen Europa. Methodik und Inhalte einer Karte im Atlas Ost- und Südosteuropa. In: Europa Regional, 7, 1, S. 2-12.

Jordan P. (2007), Aussagemöglichkeiten des Atlas Ost- und Südosteuropa im Bereich des Pannonischen Beckens. In: Hurni L., Klinghammer I., Roubitscher W. (Hrsg.), Thematische Kartierungen in den Geowissenschaften / Thematic Mapping in Geosciences. Leopoldina-Meeting vom 25. bis 27. Mai 2006 in Budapest (= Nova Acta Leopoldina, Neue Folge, 94, 349, S. 91-111. Halle (Saale), Deutsche Akademie der Naturforscher.

JoRDAN P. (2009), Mapping space-related transformation processes in Eastern Europe. Examples from the Atlas of Eastern and Southeastern Europe. In: ICA Symposium on Cartography for Central and Eastern Europe, Vienna, 16-17 February 2009, Proceedings (CD-ROM), S. 183-200.

JORDAN P. (2010), Mapping Space-Related Transformation Processes in Eastern Europe. Examples from the Atlas of Eastern and Southeastern Europe. In: Gartner G., Ortag F. (Hrsg.), Cartography in Central and Eastern Europe. Selected Papers of the $1^{\text {st }}$ ICA Symposium on Cartography for Central and Eastern Europe, S. 323-343. Berlin - Heidelberg, Springer.

Jordan P., Kelnhofer F. (1996), Der Atlas Ost- und Südosteuropa. Konzeption, Gestaltung, technische Herstellung. In: Kretschmer I., Kriz K. (Hrsg.), Kartographie in Österreich '96 (= Wiener Schriften zur Geographie und Kartographie, 9), S. 60-79. Wien, Institut für Geographie der Universität Wien, Ordinariat für Geographie und Kartographie.

Jordan P., Resch Ch., Fürpasz Ch., Kriz K., Partl F., Saul R., Riedl A. (2001), Umweltbezüge in einem webbasierten länderübergreifenden Atlas. In: Buzin R., WintgEs Th. (Hrsg.), Kartographie 2001 - multidisziplinär und multimedial. Beiträge zum 50. Deutschen Kartographentag, S. 152-163. Heidelberg, Wichmann.

KRETSCHMER I. (1995), Peter JORDAN / Fritz KELNHOFER: Atlas Ost- und Südosteuropa, 1989ff. In: Kretschmer I., Atlantes Austriaci. Österreichische Atlanten, 2. Band: 1919-1994, S. 358-359. Wien - Köln - Weimar, Böhlau.

Resch Ch., Fürpasz Ch., Jordan P., Kriz K., Partl F., Saul R., Riedl A. (2001), Characteristics in Data Management within a Scientific Multinational Internet Atlas. In: YANG K. (Hrsg.), Proceedings of the $20^{\text {th }}$ International Cartographic Conference, ICC 2001 Beijing China, August 6-10, 2001, S. 884-893. Beijing, ICA.

Saul R., Kriz K., Fürpasz Ch., Partl F., Jordan P., Resch Ch., Riedl A. (2001), Interface Design Aspects of an Interactive Atlas. In: YANG K. (Hrsg.), Proceedings of the $20^{\text {th }}$ International Cartographic Conference, ICC 2001 Beijing China, August 6-10, 2001, S. 1790-1796. Beijing, ICA.

\section{Verzeichnis der AOS-Lieferungen}

\section{1 ÖKOLOGIE - ECOLOGY}

1.1-PL1 Luftverschmutzung in Südpolen - Air Pollution in Southern Poland 1: $500.000,1: 1,000.000$

Autor - Author: Kazimierz Trafas (Kraków), erschienen - published: 1991 ISBN 3-443-28508-2 
1.2-G4 Nutzung und Probleme der Umwelt im mittleren und östlichen Europa - Use of Environment and Resultant Problems in Central and Eastern Europe 1: $3,000.000$

1.2-G4 A Umweltnutzung - Use of the Environment

1.2-G4 B Umweltprobleme - Environmental Problems

Autoren - Authors: Tat'jana Nefedova (Moskva) et al.

erschienen - published: 1992

ISBN 3-443-28509-3

1.3-M1 Topoklimatische Typen in Mitteleuropa - Topoclimatic Types in Central Europe

1: $1,500.000$

Autor - Author: Evžen QuitT (Brno)

erschienen - published: 1996

ISBN 3-443-28512-0

1.4-M2 Ökologie der Landnutzung - Ecology of Land Use in Central Europe

1: 1500.000

1.4-M2 A Landschaftseinheiten - Landscape Units

1.4-M2 B Landnutzung - Land Use

Autoren - Authors: Andrzej Richling (Warszawa) et al.

erschienen - published: 1996

ISBN 3-443-28517-1

1.5-CZ/PL1 Athropogene Reliefveränderungen in Oberschlesien - Anthropogenic Modification to the Relief of Upper Silesia

1: 200.000

Autoren - Authors: Miroslav Havrlant (Ostrava), Andrzej T. JanKowsKi (Sosnowiec)

erschienen - published: 1999

ISBN 3-443-28522-8

1.6-UA1 Boden- und Wasserqualität in der Ukraine - Soil and Water Quality in the Ukraine

1: $3,000.000$

Autoren - Authors: Leonìd G. RudenKo, Volodimir P. Razov (Kiïv) et al.

erschienen - published: 2001

ISBN 3-443-28523-6

1.7-G7 Umweltprobleme in Mittel- und Südosteuropa um 1995 - Environmental Problems in Central Southeastern Europe around 1995

1: $3,000.000$

Autoren - Authors: Arno Hartung (Leipzig), Peter Jordan (Wien), Tat‘jana Nefedova (Moskva), Joanna Plit (Warszawa) et al.

erschienen - published: 2001, 2003

ISBN 3-443-28524-4 
BEVÖLKERUNG - POPULATION

2.1-R1 Bevölkerungsverteilung in den ländlichen Gemeinden Rumäniens - Population Distribution in the Rural Communes of Romania

1: $1,500.000$

Autoren - Authors: Vasile SuRd (Cluj-Napoca), Elisabeth Tomasi (Wien), Michael

SAUBERER (Wien)

erschienen - published: 1990

ISBN 3-443-28503-1

Sprachenverteilung in Siebenbürgen - Language Distribution in Transylvania 1: 400.000

Autor - Author: Károly Kocsis (Budapest)

erschienen - published: 1990

ISBN 3-443-28506-6

2.3-YU1 Entwicklung des Hochschulwesens in Jugoslawien - Development of Higher Education in Yugoslavia

1: $3,000.000$

Autor - Author: Werner WeILGUNI (Wien/Klosterneuburg)

erschienen - published: 1991

ISBN 3-443-28507-4

2.4-PL2 Bevölkerungsentwicklung in Polen 1980-1990 - Population Development in Poland 1980-1990

1: $1,500.000$

Autor - Author: Marek KuPISZEwski (Warszawa/Leeds)

erschienen - published: 1992

ISBN 3-443-28511-2

2.5-O1 Ethnische Struktur des östlichen Europas und Kaukasiens um 1990 - Ethnic Structure of Eastern Europe and Caucasia around 1990

1: $6,000.000$

Autoren - Authors: Peter Jordan (Wien), Karl Schappelwein (Wien), Sergej TaRHov (Moskva)

erschienen - published: 1993

ISBN 3-443-28513-9

2.6-PL3 Polen als Quelle von Wanderungen und Reisen - Poland as a Source of Migration and Travel

1: $3,000.000,1: 20,000.000$

Autor - Author: Marek KuPISZEwski (Warszawa/Leeds)

erschienen - published: 1993

ISBN 3-443-28514-7 
2.7-S1 Ethnische Struktur Südosteuropas um 1992 - Ethnic Structure of Southeastern Europe around 1992

1: $1,500.000$

Autoren - Authors: Peter JoRdan (Wien), Gešo GešEv (Sofija) et al. erschienen - published: 1995

ISBN 3-443-28516-3

2.8-H/RYU1 Entwicklung der ethnischen Struktur des Banats 1890-1992 - Development of Ethnic Structure in the Banat 1890-1992

1: 400.000

2.8-H/RYU1 A: 1890

2.8-H/RYU1 B: um 1930 - around 1930

2.8-H/RYU1 C: um 1950 - around 1950

2.8-H/RYU1 D: um 1990 - around 1990

Autoren - Authors: Josef Wolf (Tübingen), Horst Förster (Tübingen)

erschienen - published: 1999, 2004

ISBN 3-443-28519-3

2.9-G9 Ethnisches Bewusstsein in Mittel- und Südosteuropa um 2000 - Ethnic Consciousness in Central and Southeast Europe around 2000

1: $3,000.000$

Autoren - Authors: Peter JoRdan (Wien), Károly Kocsis (Budapest) et al.

erschienen - published: 2006, 2007

ISBN 3-443-28516-3

2.10-MD1 Ethnisches Bewusstsein in der Republik Moldau im Jahr 2004 - Ethnic Consciousness in the Republic of Moldova in 2004

1: 600.000

Autoren - Authors: Thede KAHL (Jena), Dorin Lozovanu (Chişinău), Valeria HeuBERGER (Wien)

erschienen - published: 2009

ISBN 3-443-28529-5

3

WIRTSCHAFT - ECONOMY

3.1-H1 Der Aufschwung des Fremdenverkehrs aus westlichen Ländern nach Ungarn in den 80er Jahren - The Expansion of Tourism from Western Countries to Hungary in the Eighties

1: $1,250.000 ; 1: 2,500.000$

Autoren - Authors: Peter Jordan (Wien), György Miczek (Budapest)

erschienen - published: 1989

ISBN 3-443-28502-3

3.2-G2 Energiewirtschaft Ost- und Südosteuropa - Power Industry of Eastern and Southeastern Europe

1: $3,000.000$

Autoren - Authors: Karl Schappelwein (Wien), Hubert Gabrisch (Wien)

erschienen - published: 1990

ISBN 3-443-28505-8 
Erste sozioökonomische Effekte der Transformation im mittleren und östlichen Europa - First Socio-economic Effects of Transformation of Central and Eastern Europe

1: $6,000.000$

Autoren - Authors: Peter Jordan (Wien), Tat'jana Nefedova (Moskva) et al. erschienen - published: 1994

ISBN 3-443-28515-5

3.4-G6 Internationale Tourismusattraktionen in Mittel- und Südosteuropa - International Tourism Attractions in Central and Southeastern Europe 1: $3,000.000$

Autoren - Authors: Peter Jordan (Wien), Karl Schappelwein (Wien) et al. erschienen - published: 1999

ISBN 3-443-28521-X

Transformation der Landwirtschaft in Mittel- und Südosteuropa - Transformation of Agriculture in Central and Southeast Europe

1: $6,000.000$

Autoren - Authors: Elke KnAPPE (Leipzig), Marina RatČIna (Leipzig) et al.

erschienen - published: 2004

ISBN 3-443-285215-2

3.6-G11 Wiener Direktinvestitionen in Mittel- und Südosteuropa - Foreign Direct Investment from Vienna in Central and Southeast Europe

1: $6,000.000$

Autoren - Authors: Robert MusiL (Wien) et al.

erschienen - published: 2011

ISBN 3-443-285531-9

VERKEHR - TRAFFIC

5

RAUMPLANUNG, RAUMENTWICKLUNG - SPATIAL PLANNING, SPATIAL DEVELOPMENT

5.1-G1 Verwaltungsgliederung Ost- und Südosteuropas - Administrative Subdivision of Eastern and Southeastern Europe

1: $3,000.000$

Autoren - Authors: Peter JoRdan (Wien), Ilona SLAWINSKI (Wien)

erschienen - published: 1989

ISBN 3-443-28501-5

5.2-R2 Ausstattung der ländlichen Siedlungen in Siebenbürgen mit zentralen Einrichtungen - Availability of Central Facilities in Rural Settlements of Transylvania

1: 400.000

Autoren - Authors: Vasile Surd (Cluj-Napoca), Elisabeth Tomasi (Wien), Michael

SAUberer (Wien)

erschienen - published: 1990

ISBN 3-443-28504-X 
5.3-MO1 Zentrensysteme in Mittel- und Osteuropa - Central Place Systems in Central and Eastern Europe

1: $3,000.000$

Autoren - Authors: Frank-Dieter Grimm (Leipzig) et al. erschienen - published: 1997

ISBN 3-443-28518-X

5.4-G10 Verwaltungsgliederung Mittel- und Südosteuropas 2007 - Administrative Subdivision of Central and Southeast Europe 2007

1: $3,000.000$

Autoren - Authors: Peter Jordan (Wien), Florian PARTL (Wien)

erschienen - published: 2010

ISBN 3-443-28527-9

6

SONSTIGE - OTHERS

Die Wahlen des Jahres 1990 in Mittel-, Ost- und Südosteuropa - The Elections of 1990 in Central, Eastern and Southeastern Europe

1: $3,000.000$

Autoren - Authors: Peter Jordan (Wien), Karl Schappelwein (Wien) et al.

erschienen - published: 1991

ISBN 3-443-28510-4

6.2-G5 Die Wahlen der Jahre 1994-1997 in Mittel-, und Südosteuropa - The Elections of 1994-1997 in Central and Southeastern Europe

1: $3,000.000$

Autoren - Authors: Peter JoRdAn (Wien), Gerhard MangotT (Wien), Karl SchaPPELWEIN (Wien) et al.

erschienen - published: 1998

ISBN 3-443-28520-1

Präsidentenwahlen 2009 in Rumänien - Presidential Elections 2009 in Romania 1: $1,500.000$

Autoren - Authors: Leonard Bruckner (Cluj-Napoca), Adina Puscasu (ClujNapoca), Voicu Bodocan (Cluj-Napoca)

erschienen - published: 2014

ISBN 3-443-28532-6 